\title{
High-Speed Rainbow Schlieren Visualization of an Oscillating Helium Jet Undergoing Gravitational Change
}

\author{
Peter A. Leptuch ${ }^{* 1}$ and Ajay K. Agrawal ${ }^{* 2}$ \\ *1 Graduate Research Assistant, School of Aerospace and Mechanical Engineering, University of \\ Oklahoma, Norman, OK 73019, USA \\ *2 Lloyd G. \& Joyce Austin Presidential Professor and Associate Professor, School of Aerospace \\ and Mechanical Engineering, University of Oklahoma, Norman, OK 73019, USA, email: \\ aagrawal@ou.edu
}

\begin{abstract}
Rainbow schlieren deflectometry combined with high-speed digital imaging was used to study buoyancy effects on flow structure of a helium jet discharged vertically into air. The experimental data were taken using the 2.2 -sec drop tower facility at the NASA John H. Glenn Research Center in Cleveland, Ohio. The test conditions pertained to jet Reynolds number of 490 and jet Richardson number of 0.11, for which buoyancy is often considered unimportant. Experimental results show global oscillations at a frequency of $27 \mathrm{~Hz}$ in Earth gravity. In microgravity, the jet oscillations vanished and the jet width increased. Results provide a direct physical evidence of the importance of buoyancy on the flow structure of lowdensity gas jets at a Richardson number considered too small to account for gravity.
\end{abstract}

Keywords: Color Schlieren, Low-Density Jets, Flow Oscillations, High-Speed Imaging

\section{Introduction}

Flow structure of gas jets has been extensively investigated because of their wide-ranging applications. An important focus of these studies involves the formation of instabilities in the near field and the evolution of developed structures in the downstream region. Some of these studies have used the concept of introducing flow oscillations in the jet tube, which are examined subsequently in the downstream flow field. In contrast, the present study considers a flow configuration with oscillations occurring naturally in the Earth gravity environment. It consists of a low-density gas jet (helium) injected into a high-density atmosphere (air). Self-excited oscillations in low-density jets have been observed for jet Reynolds numbers, $\operatorname{Re}\left[=U_{j} d / v_{j}\right.$, where $U_{j}$ is the average jet velocity, $\mathrm{d}$ is the tube inside diameter, and $v_{j}$ is kinematic viscosity of the jet fluid] varying from a few hundreds (Hamins et al, 1992; Subbarao and Cantwell, 1992; Richards et al, 1996; Cetegen, 1997) to several thousands (Monkewitz et al, 1990; Kyle and Sreenivasan, 1992). The instability is found to produce distinctly periodic oscillations in the near field, leading to an early and abrupt breakdown of the potential core. The jet is characterized as momentum-dominated for Richardson number, $\mathrm{Ri}<<1.0$, where $\mathrm{Ri}=\operatorname{gd}\left(\rho_{a}-\rho_{j}\right) / \rho_{j} U_{j}^{2} ; \mathrm{g}$ is the gravitational acceleration, $\rho_{a}$ and $\rho_{j}$ are, respectively, the ambient and jet densities. The jet is considered buoyant for $\mathrm{Ri}>1$, although buoyancy may still be important at lower Ri.

Subbarao and Cantwell (1992) observed highly periodic oscillations in the entire near field of helium jets issued in a co-flow of air for Ri varying between 0.5 and 6.0. The oscillation frequency (f) represented by Strouhal number, $\mathrm{St}=[\mathrm{fd} / \mathrm{Uj}]$ correlated with Richardson number for $\mathrm{Ri} \geq 1.0$, indicating a buoyancy-dependent instability mode. For $\mathrm{Ri}<0.5$, the Strouhal number was independent of the Richardson number and hence, the flow oscillations were considered to be independent of the gravity. The buoyancy was also considered negligible in momentum-dominated low-density jets of 
Kyle and Sreenivasan (1993) at $\mathrm{Ri}$ on the order of $10^{-3}$ and those of Richards et al (1996) for $\mathrm{Ri}=0.3$ to $8^{*} 10^{-3}$, bridging the buoyancy-dominated to momentum-dominated jet flow regimes. Evidently, origin of the instability in low-density gas jets leading to global oscillations in the near field has not been established for $\mathrm{Ri}<0.5$. Specifically, the role of gravity has not been delineated in this flow regime.

The above studies conducted in Earth gravity pose experimental limitations in isolating buoyancy effects on the flow structure of low-density jets. First, the experimental variables, i.e., Reynolds number and Richardson number depend upon each other, and therefore, they cannot be varied independently. Subbarao and Cantwell (1992) controlled the operating pressure to circumvent this problem, although for a narrow range of operating conditions. Secondly, even though the buoyancy may be rendered negligible globally in a momentum-dominated jet, it cannot be eliminated. Buoyancy may be significant locally in the low-momentum regions of the flow, e.g., the jet shear layer and thereby, affect the jet instability leading to significant changes in the flow field. Eliminating buoyancy throughout the flow field requires experiments in zero or microgravity environment, which also provides the flexibility to vary the Richardson number by several orders of magnitude for a given Reynolds number.

Yep et al (2003) used the microgravity environment of a drop tower to show that the flow oscillations in a low-density jet in Earth gravity at $\mathrm{Ri}=1.44$ were buoyancy induced. Self-excited oscillations in Earth-gravity disappeared as the jet flow adjusted to microgravity conditions in the drop tower. They also provided quantitative details of buoyancy-induced changes in the helium concentration field using the rainbow schlieren deflectometry technique. The present study extends the investigation by Yep et al. (2003) to a self-excited low-density jet in Earth gravity at a smaller Richardson number $(\mathrm{Ri}=0.11)$, for which the origin of the instability has not yet been established. The $60 \mathrm{~Hz}$ imaging system used by Yep et al (2003) was incapable of resolving the high oscillation frequencies observed at low Richardson numbers. Consequently, the rainbow schlieren apparatus was modified by integrating it with a high-speed digital imaging system, and used for the first time in the $2.2^{-\mathrm{s}}$ drop tower to visualize and quantify effects of gravity in a low-density jet bridging the buoyancy dominated and momentum-dominated flow regimes. Unlike Yep et al (2003), the present experiment however did not seek to quantify the concentration field.

\section{Experimental Approach}

The experiment was conducted at the NASA John H. Glenn Research Center's 2.2s drop tower facility. Upon release, the experimental package undergoes free fall in the drop tower and experiences microgravity after an initial period of $0.1 \mathrm{~s}$ during which the acceleration decreases linearly from Earth gravity to microgravity (Lekan, 2004). The drop rig shown in Figure 1 was a battery powered testing device contained within a sturdy aluminum frame of approximate dimensions: $0.84 \mathrm{~m}$ high, $0.97 \mathrm{~m}$ wide and $0.41 \mathrm{~m}$ deep. A thick curtain covering the rig isolated the jet flow from external disturbances. All aspects of system design were constrained to withstand impact decelerations of up to $100 \mathrm{~g}$. The flow system comprised of the following components: 1) two compressed gas reservoirs with helium stored at a pressure of 14bar, 2) a mass flowmeter calibrated for helium in the range of 0.0 to 60.0 standard liters per minute, 3) a control valve to specify the helium gas flow rate, 4) a solenoid valve to instantaneously initiate and terminate the flow, 5) a heavy duty, clear, plastic, flexible hose, filled with steel wool to stabilize the flow, 6) a U-tube segment to direct the flow from the flexible tube to the inlet of a straight jet tube, and 7) the straight, polished, jet tube to vertically discharge the flow into quiescent air. The length and diameter of the jet tube were, respectively, $300-\mathrm{mm}$ and $18.7-\mathrm{mm}$. Because of the spatial constraints of the drop rig, the tube length was insufficient to attain fully-developed laminar flow conditions at the exit.

The components of the rainbow schlieren deflectometry system, positioned on an optical breadboard, included 1) the light source aperture ( $3 \mathrm{~mm}$ high and $0.1 \mathrm{~mm}$ wide) connected through an optical fiber cable to an external $160 \mathrm{~W}$ halogen light source, 2) the collimating lens $(80 \mathrm{~mm}$ diameter with focal point $310 \mathrm{~mm}$ upstream at the source aperture), 3) the decollimating lens (80mm diameter 
with focal point $1000 \mathrm{~mm}$ downstream at the filter plane), 4) two aluminum coated, flat surface, reflecting mirrors, 5) a $3.5 \mathrm{~mm}$ symmetric rainbow filter positioned at the focal point of the decollimating lens and 7) the camera lens, sensor, and data storage. A high-speed digital camera, model Motion Xtra HG-TX by Redlake, was employed to capture and digitally store color schlieren images at exposure time of 663- $\mu$ s and acquisition rate of 1000 frames per second.

The test sequence consisted of 1) activating the solenoid valve to turn on the flow in Earth gravity, 2) allowing time to establish the oscillating flow, 3) triggering storage of digital schlieren images in the on-board camera memory, 4) initiating the drop, 5) deactivating the solenoid valve to turn-off the flow, 6) retrieving the rig, and (7) transferring image data from camera memory to the hard disk media. The drop procedure was automated using a programmable logic controller (PLC). The image date were processed to (1) create time-space images to depict temporal evolution of the flow at specified axial locations, (2) determine the oscillation frequency at various locations using fast Fourier analysis, and (3) generate hue distributions to study detailed flow structure of the jet.

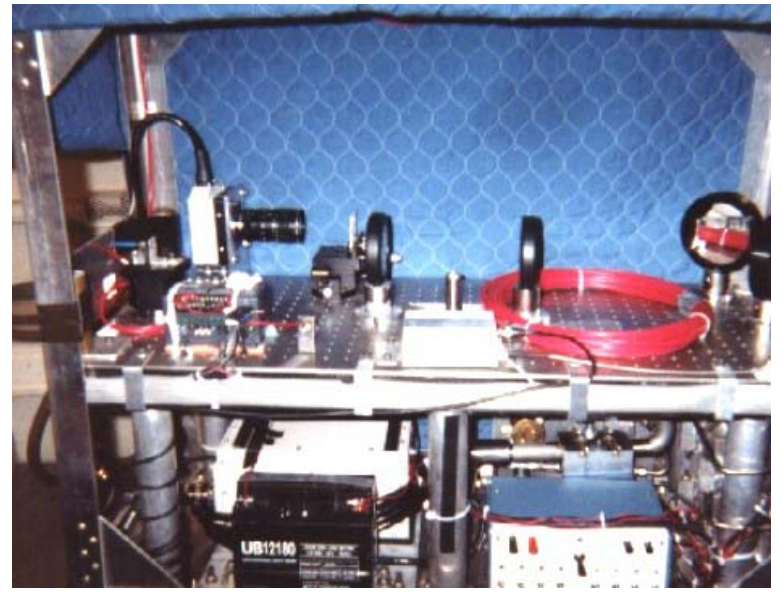

Figure 1. Photograph of the drop rig. Clearly A two-level design with the flow components and power system on the bottom and the optical system on the upper level was used. A battery and the camera controller body are visible on the lower level, while the fuse box and some piping components are visible on the bottom right. The camera lens, mirrors, collimating and decollimating lenses, test section, light source, and PLC are visible on the upper level. Note that a fiber optic cable for light input is coiled and taped in place on the upper floor of the drop rig. It would be connected to an external light source during the drop.

\section{Results and Discussion}

\subsection{Rainbow Schlieren Images}

The test conditions reported in this study pertain to jet Reynolds number of 490 and jet Richardson number of 0.11. Figure 2 shows schlieren images at various phases to depict an oscillation cycle in Earth gravity. The jet tube is visible as the black region at the bottom of each image. Figure 2 shows the primary jet bulging near the exit and contracting gradually in the downstream direction, where a separate outer structure is observed. The region between primary jet and outer flow, signifying a toroidal structure, moves downstream during the oscillation cycle. Concurrently, the primary jet contracts near the exit and separates from the outer flow to create a new toroidal structure. Thereafter, a new bulge develops near the jet exit as the oscillation cycle repeats itself. The flow structure observed in Fig. 2 was found to be highly repeatable. The oscillating flow in Earth gravity undergoes a significant change in microgravity. Figure 3 shows a sequence of schlieren images at 20$\mathrm{ms}$ interval, beginning with the onset of free fall. Evidently, the flow oscillations continue for a brief period, as the outer toroidal structure formed in Earth gravity is swept downstream. 


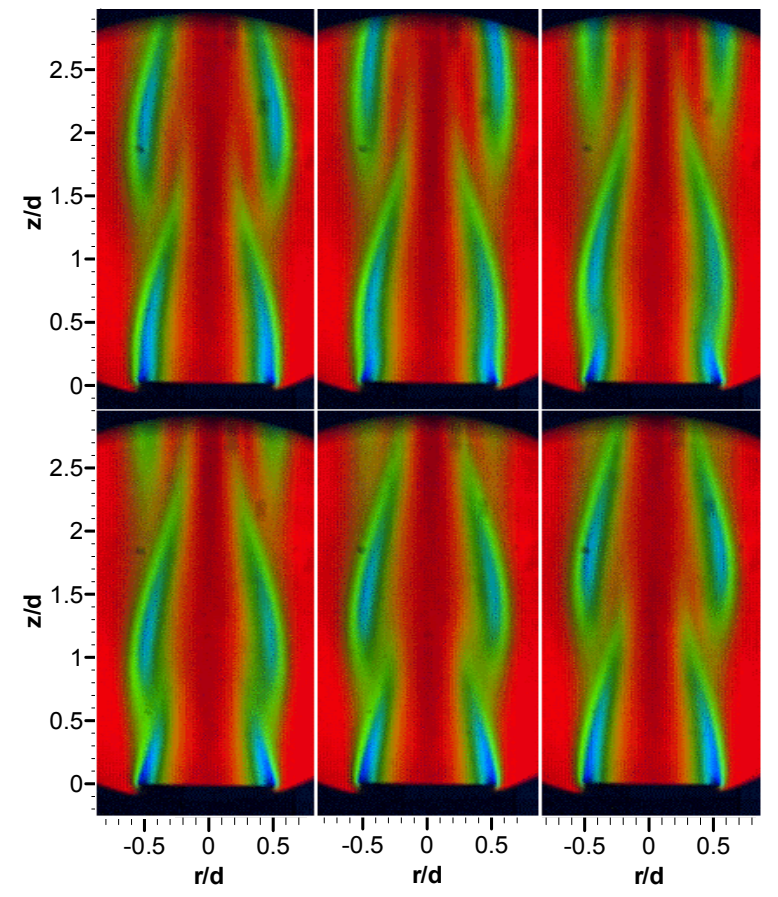

Figure 2. Rainbow schlieren image to depict a typical oscillation cycle in Earth gravity. Images progress at $7 \mathrm{~ms}$ interval, from left to right starting in the upper left corner.

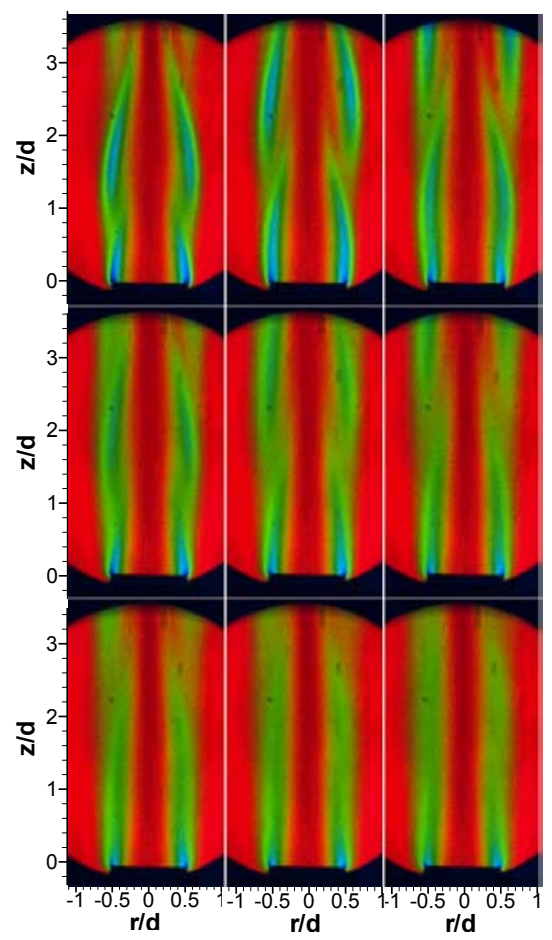

Figure 3. Rainbow schlieren images depicting flow structure during change from Earth gravity to microgravity. The images begin at the onset of free fall and continue from left to right, top to bottom at $20 \mathrm{~ms}$ interval. 
Periodic bulging and contacting of the primary jet leading to the formation of the outer toroidal structure in Earth gravity is absent in microgravity. Results show that the flow approaches steady conditions after a brief period of free fall.

\subsection{Space-Time Images}

Although nearly 2700 color schlieren images were obtained in the experiment, only a few are shown in Figs. 2 and 3. Thus, schlieren data from successive images were concatenated to create space-time images at selected axial locations. Figure 4 shows a portion of space-time images constructed from 700 schlieren images to span about $700 \mathrm{~ms} ; 260 \mathrm{~ms}$ in Earth gravity and $440 \mathrm{~ms}$ in free fall. These images are shown for $\mathrm{z} / \mathrm{d}=0.01,0.5,1.0,1.5$, and 2.5 , where $\mathrm{z}$ represents the distance downstream of the tube exit.

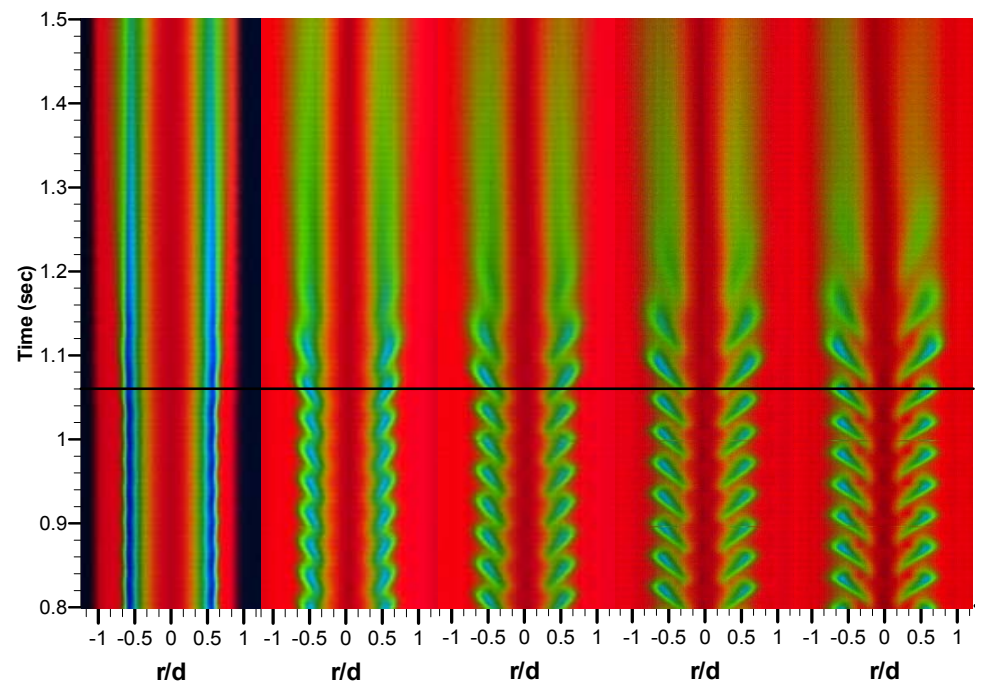

Figure 4. Space-time images at $\mathrm{z} / \mathrm{d}=$ (a) 0.01 , (b) 0.5, (c) 1.0, (d) 1.5, and (e) 2.5, from left to right, respectively. The black line at $\mathrm{t}=1.06-\mathrm{s}$ indicates start of microgravity.

The horizontal black line in each image denotes the approximate release time $(t=1.06 \mathrm{~s})$ of the experiment in the drop tower. Thus, the jet flow experiences Earth gravity for $t \leq 1.06 \mathrm{~s}$ and free fall for $\mathrm{t}>1.06 \mathrm{~s}$. Figure 4 provides detailed visualization of how the jet flow evolves temporally and spatially. In Earth gravity, the flow oscillations are observed at all axial locations shown in Fig. 4. The oscillation amplitude at $\mathrm{z} / \mathrm{d}=0.01$ is small (discernable upon magnification) and it increases with downstream locations. Oscillations penetrated farther into the jet center region at downstream locations. The wavy structure at $\mathrm{z} / \mathrm{d}=0.5$ represents the cyclic bulging and contracting of the primary jet. However, at $\mathrm{z} / \mathrm{d}=1.0$ and further downstream, the primary jet appears to separate from the outer flow to signify the presence of an outer toroidal structure. Figure 4 reveals a highly repeatable flow structure between consecutive cycles in Earth gravity. At all axial locations, about 7 cycles are completed in $260 \mathrm{~ms}$ duration of Earth gravity suggesting that the flow oscillates globally at a frequency of approximately $27 \mathrm{~Hz}$. The resulting Strahoul number of 0.16 is the same as that obtained from the correlation of Cetegen and Kasper [1996] i.e., $S t=0.8\left[R i \rho_{a} / \rho_{j}\right]^{0.38}$.

The image portion above the black line in Fig. 4 represents temporal evolution of the flow structure during change from Earth gravity to microgravity in the drop tower. Results show oscillations persisting during a brief initial period of free fall. The increased time between successive structures at the onset of free fall signifies a decrease in the oscillation frequency. The decay in jet oscillations appears to start from the jet exit and works it way downstream. For example, at $\mathrm{t}=1.2 \mathrm{~s}$, 
oscillating structure is observed at $\mathrm{z} / \mathrm{d}=2.5$ although the flow at $\mathrm{z} / \mathrm{d}=0.5$ appears steady. The flow oscillations at all locations have seized after about $200 \mathrm{~ms}$ of free fall or $\mathrm{t}=1.25 \mathrm{~s}$. Two factors account for the persistence of oscillations after the drop is initiated; the time required for attaining microgravity conditions in the drop tower and the flow adjustment time. The microgravity conditions are reached after $100 \mathrm{~ms}$ of free fall [Lekan, 2004] and the flow convection time based on the average jet exit velocity is about $15 \mathrm{~ms}$. Thus, oscillations continue mainly because of the finite gravitational level existing during the initial period of free fall. Results show that the jet in microgravity is wider than that in Earth gravity. Lack of buoyancy in microgravity causes the jet to spread mainly by the molecular diffusion. Accordingly, the density varies gradually to produce smaller angular deflections in the radial direction, reflected by fewer color gradations in schlieren images in microgravity. Results in Fig 4 provide direct physical evidence that the flow oscillations in Earth gravity are buoyancy induced.

\subsection{Fast Fourier Transform (FFT) Analysis}

In this section, an analysis of the power spectra of the flow field is presented. The analysis is based on Fast Fourier Transform (FFT) of hue values at a specified point in the flow field, obtained from 512 successive (in time) images, at a resolution of $1 \mathrm{~Hz}$. Three partially overlapping 512-image subsections of data were used in the FFT analyses to represent the early, mid, and late duration of microgravity. Figure 5 shows the power spectra at $\mathrm{z} / \mathrm{d}=0.5$ and 1.5 for $\mathrm{r} / \mathrm{d}=0.5$.

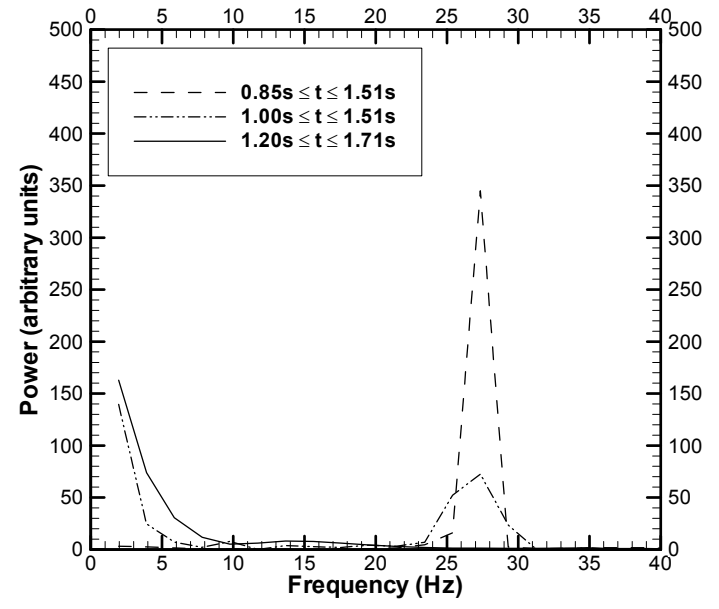

Figure 5a. Power spectra at $\mathrm{r} / \mathrm{d}=0.5, \mathrm{z} / \mathrm{d}=0.5$.

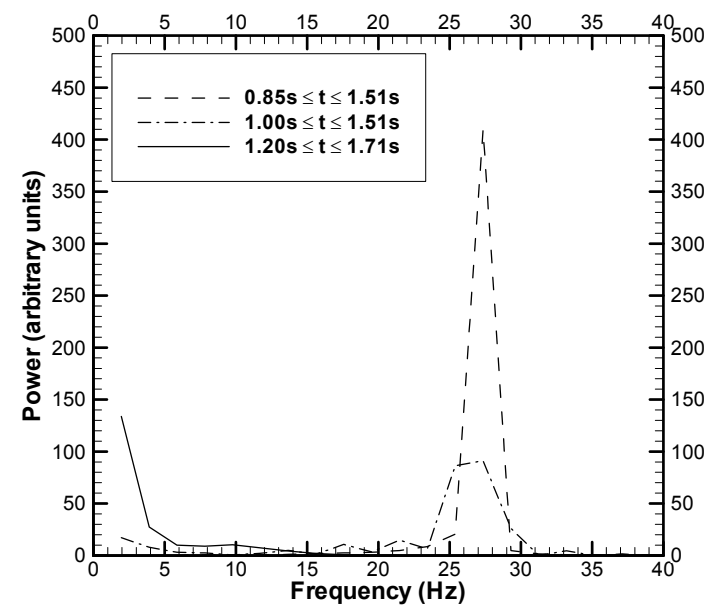

Figure 5b. Power Spectra at $\mathrm{r} / \mathrm{d}=0.5, \mathrm{z} / \mathrm{d}=1.5$.

In Fig. 5a, a characteristic peak in the power spectra is observed at a frequency of $27 \mathrm{~Hz}$ for the "early" period $0.85 \mathrm{~s} \leq \mathrm{t} \leq 1.51 \mathrm{~s}$, where the free fall begins at $\mathrm{t}=1.06 \mathrm{~s}$ and microgravity conditions are reached at $\mathrm{t}=1.16 \mathrm{~s}$. Evidently, the FFT analysis captures the dominant frequency of Earth gravity oscillations persisting during the early microgravity period. Note that Earth gravity oscillation frequency of approximately $27 \mathrm{~Hz}$ was also determined from visual observations in the previous section. The curve for the "mid" period $1.00 \mathrm{~s} \leq \mathrm{t} \leq 1.51 \mathrm{~s}$ in Fig $5 \mathrm{a}$ also shows a peak near $27 \mathrm{~Hz}$, signifying Earth gravity as the source of oscillations. A peak is also observed near $0 \mathrm{~Hz}$. The $27 \mathrm{~Hz}$ peak has disappeared and the $0 \mathrm{~Hz}$ peak has intensified for the "late" period $1.20 \mathrm{~s} \leq \mathrm{t} \leq 1.71 \mathrm{~s}$ spanning only the microgravity data. Similar results are obtained at a downstream location $(\mathrm{z} / \mathrm{d}=1.5)$ as shown in Fig. 5b. Clearly, the self-excited oscillations in Earth gravity diminish in microgravity. 


\subsection{Hue Distribution Plots}

Plots of hue distribution at various axial planes were generated to show temporal evolution of the flow structure. In a rainbow schlieren apparatus, the change in hue represents the angular deflection of light rays caused by density gradients in the flow field (Alammar et al., 1998). Hue profiles at $\mathrm{z} / \mathrm{d}=0.5$ and 1.5 in Fig. 6 show that the oscillation amplitude in Earth gravity increases in the axial direction. A constant oscillation frequency of $27 \mathrm{~Hz}$ was obtained by measuring the time between peaks and valleys of various hue contour levels in Fig. 6. The measured oscillation frequency matches with that computed using the FFT analysis. In agreement with results presented earlier, hue oscillations subside in microgravity. The outer edges of the schlieren image provide direct measure of the jet width based on the concentration field. The jet width thus obtained is approximately twice of

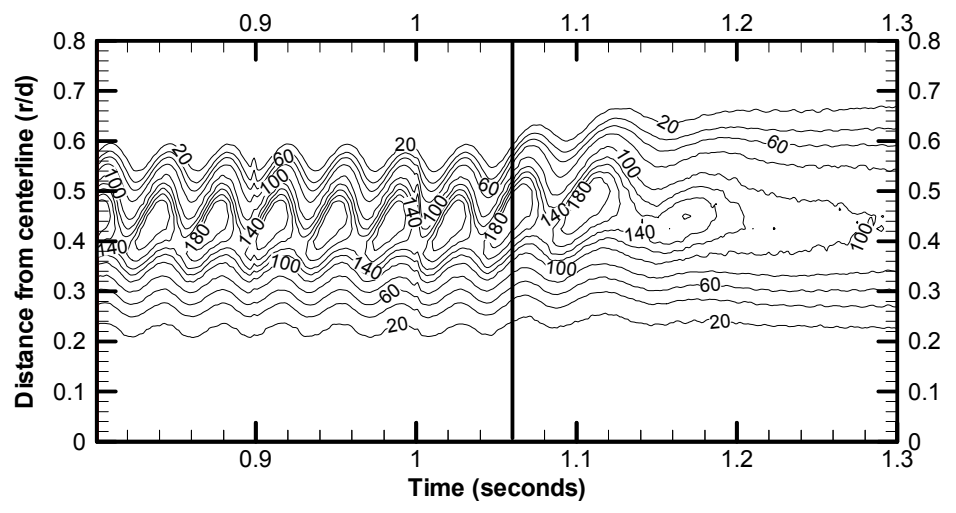

the jet half width (related to the radial location where the velocity or concentration value is half of that at the centerline) typically reported in the literature. In this experiment, the schlieren boundary is approximated by the hue value of 20 degrees, which is close to the background hue of the color schlieren image. Figure 6 shows that the outer edge of the jet (or the 20 degree hue contour) in Earth gravity oscillated between $\mathrm{r} / \mathrm{d}$ of 0.54 and 0.60 . In microgravity, the jet outer edge was located at $\mathrm{r} / \mathrm{d}=0.67$, signifying an increase of jet width in microgravity. Similarly, at $\mathrm{z} / \mathrm{d}=1.5$, the jet outer edge in Earth gravity oscillated between $\mathrm{r} / \mathrm{d}$ of 0.52 and 0.64 and it was located approximately at $\mathrm{r} / \mathrm{d}=0.67$ in microgravity. These results concur with the earlier evidence for buoyancy-dominated jets (Yep et al., 2003) showing oscillations decaying and jets widening as microgravity conditions are reached.

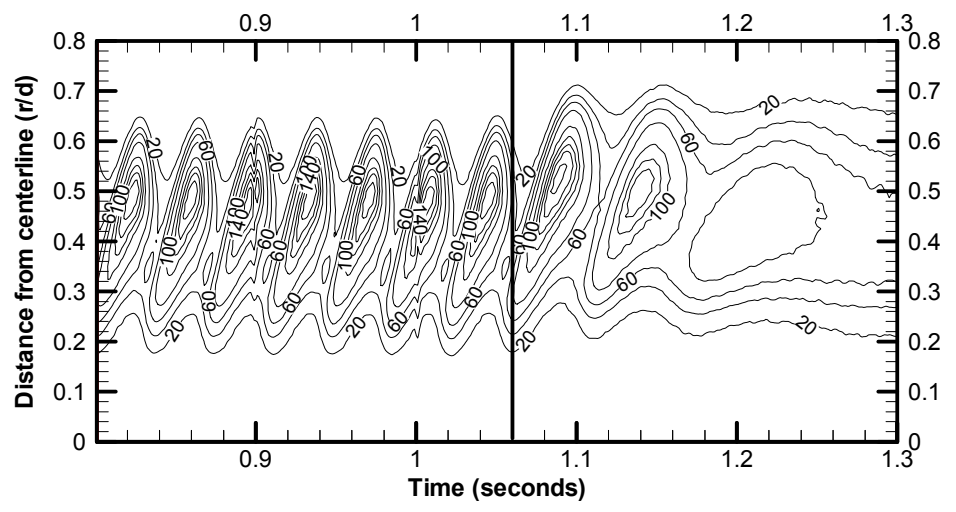

Figure $6 \mathrm{~b}$. Hue profiles at $\mathrm{z} / \mathrm{d}=1.5$. 


\subsection{Conclusions}

High-speed rainbow schlieren deflectometry technique was used to visualize and quantify buoyancy effects on the flow fields of a helium jet discharged vertically into ambient air at a Richardson number of 0.11 , bridging the buoyancy-dominated and momentum-dominated flow regimes. Flow structure details were investigated using space-time images, power spectra and profiles of hue obtained from rainbow schlieren images. Results show highly periodic oscillations in the Earth gravity. A constant oscillation frequency of $27 \mathrm{~Hz}$ was obtained throughout the flow field using different analysis techniques. Earth gravity oscillations subsided after a brief period of free fall during which the gravitational level decreased from Earth gravity to microgravity. The jet width increased in microgravity. Results provide direct physical evidence that the flow oscillations at the small Richardson number considered in this study were buoyancy induced. Results suggest that the Richardson number based on global parameters is not sufficient to fully characterize buoyancy effects in low-density gas jets. Finally, high-speed rainbow schlieren apparatus was developed and demonstrated by obtaining temporally and spatially resolved measurements in the microgravity environment of the drop tower.

\section{Acknowledgements}

This work was supported by the Physical Sciences Division of NASA's Office of Biological and Physical research under grant NAG 3-2388.

\section{References}

Al-Ammar, K., Agrawal, A.K., Gollahalli, S.R., and Griffin, D.W., 1998, "Application of Rainbow Schlieren Deflectometry for Concentration Measurements in an Axisymmetric Helium Jet," Exp. Fluids, vol. 25, 89.

Cetegen, B.M., and Kasper, K.D., 1996, "Experiments on the Oscillatory Behavior of Buoyant Plumes of Helium and Helium-Air Mixtures,” Physics of Fluids, vol. 8, 2974.

Cetegen, B. M., 1997 "Behavior of Naturally Unstable and Periodically Forced Axisymmetric Buoyant Plumes of Helium and Helium-Air Mixtures," Phys. Fluids, vol. 9, 1.

Greenberg, P.S., R.B. Klimek and D. R. Buchele, 1995, "Quantitative Rainbow Schlieren Deflectometry," App. Opt., vol. 34, 3819 .

Hamins, A., Yang, J.C., and Kashiwagi, T., 1992, "An Experimental Investigation of the Pulsating Frequency of Flames," Proc. Combust. Inst., vol. 24, 1695.

Kyle D. M. and K. R. Sreenivasan, 1993, "The Instability and Breakdown of a Round Variable-Density Jet," J. Fluid Mech., vol. 249, 621.

Lekan, J.H, 2004, Private Communication, NASA Glenn Research Center.

Monkewitz, P.A., Bechert, D.W., Barsikow, B., and Lehmann, B., 1990, "Self-Excited Oscillations and Mixing in a Heated Round Jet,” J. Fluid Mech., vol. 213, 611.

Richards, C.D., Breuel, B.D., Clark, R.P., and Troutt, T.R., 1995, "Concentration Measurements in a Self-Excited Jet," Exp. Fluids, vol. 21, 103.

Subbarao, E. R., and B. J. Cantwell, 1992, "Investigation of a Co Flowing Buoyant Jet: Experiments on the Effect of Reynolds Number and Richardson Number," J. Fluid Mech., vol. 245, 69.

Yep, T.W., Agrawal, A.K., and Griffin, D.W., 2003, "Gravitational Effects on Near Field Flow Structure of LowDensity Jets," AIAA Journal, vol. 41, 1973. 


\section{Authors Profile}

Peter A. Leptuch: He received his Master of Science degree in Mechanical Engineering in 2002 and Bachelor of Science degree in Meteorology in 1998; both from the University of Oklahoma. His research interests include fluid flows in microgravity and software development.

Ajay K. Agrawal: He is Lloyd G. \& Joyce Austin Presidential Professor and Associate Professor in the School of Aerospace and Mechanical Engineering at the University of Oklahoma. Professor Agrawal received his doctoral degree from the University of Miami in 1988. Prior to joining University of Oklahoma in 1993, he was visiting faculty at Michigan Technological University (1988-89) and Clemson University (1989-1993). His research interests include flow and combustion in microgravity, and environmentally benign energy utilization. 